\title{
Unusual case of abdominal wall pseudohernia in a patient with acute constipation
}

Tae Hee Lee

Institute for Digestive Research, Digestive Disease Center, Soonchunhyang University Seoul Hospital, Seoul, Korea
Received: March 21, 2020

Revised : April 1, 2020

Accepted: April 2, 2020

\section{Correspondence to}

Tae Hee Lee, M.D.

Tel: +82-2-710-3084

Fax: +82-2-709-9696

E-mail: imano825@schmc.ac.kr

https://orcid.org/0000-0003-

3049-8252
A 56-year-old man presented with a protrusion from the right abdominal wall (Fig. 1A) and acute-onset constipation. Three weeks prior, he had been diagnosed with herpes zoster of the T9-11 dermatome on the right side in an independent dermatology clinic, for which he had received oral famciclovir, pregabalin, and acetaminophen. When the patient visited our Hernia Center and Department of Gastroenterology, he denied having symptoms of bladder dysfunction. He had previously had a regular bowel habit. $\mathrm{He}$ stated that the bulging on the right side of his abdominal wall occurred following excessive straining during defecation. This abdominal bulge became more prominent upon standing or execution of the Valsalva maneuver. A physical examination revealed multiple rashes distributed unilaterally accompanied by a hard, overlying crust (Fig. 1B). The lesions did not exceed the midline. The patient's laboratory findings were unremarkable. Abdominal computed tomography showed dilation of the proximal colon with a concomitant protrusion on the right side of the abdominal wall (Fig. 1C). A diagnosis of segmental motor paresis with coexisting visceral neuropathy was made. The patient was treated with analgesics and combinations of laxatives such as polyethylene glycol 4000 (Forlax, Beaufour Ipsen Pharm Co., Paris, France), prucalopride, pyridostigmine, and misoprostol. These medications ameliorated the severe constipation. At follow-up 2 months later, the distention of the right abdominal wall remained apparent, but the constipation was relieved.

Herpes zoster occurs predominantly in the thoracic area. However, the incidence of zoster motor paresis in this area, which presents with pseudohernia of the abdominal wall, is low. Zoster abdominal paresis is occasionally accompanied by visceral neuropathy,

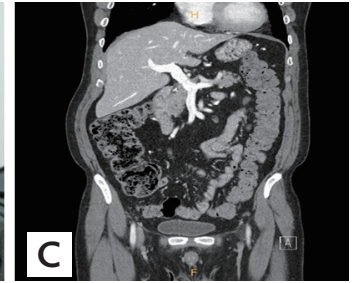

Figure 1. Physical examination and radiological findings. (A) Protrusion of the right abdominal wall 3 weeks after the onset of herpes zoster rash. (B) Multiple skin rashes accompanying a hard, overlying crust. (C) Abdomen-pelvis computed tomography showing convexity of the right abdominal wall without a defect in the abdominal wall or fascia and segmental dilation of the proximal colon, compared to the distal colon. 
which can lead to gastrointestinal complications such as intestinal pseudo-obstruction and constipation. Here, we describe an unusual case of abdominal segmental hernia and acute-onset constipation in a patient with herpes zoster. The patient provided informed consent for publication.

\section{Conflict of interest}

No potential conflict of interest relevant to this article was reported.

\section{Acknowledgments}

This work was supported by the Soonchunhyang University Research Fund. 